\title{
Exito reproductivo de plantas ornitócoras en un relicto de selva subtropical en Argentina
}

\author{
Reproductive success of bird-dispersed plants in a subtropical forest relict in Argentina
}

NORBERTO H. MONTALDO

Cátedra de Botánica, Departamento de Ecología. Facultad de Agronomía de la Universidad de Buenos Aires. Av. San Martín 4453, 1417 Buenos Aires, Argentina, e-mail: montaldo@mail.agro.uba.ar

\begin{abstract}
RESUMEN
En un relicto de selva ribereña situado en la región central de Argentina (Punta Lara, provincia de Buenos Aires) se estudió la fenología reproductiva, y la producción y dispersión de diásporas de cinco especies arbóreas nativas y de dos malezas exóticas (Rubus ulmifolius y Ligustrum lucidum). Además se determinó el poder germinativo de las semillas y la tasa de reclutamiento y características de las plántulas. En el último medio siglo las malezas invadieron la selva, amenazando actualmente con destruirla. El elenco de aves frugívoras del área es reducido y está integrado por siete especies residentes y una migratoria. Hay plantas nativas que manifiestan limitaciones reproductivas por escasa producción y/o dispersión de diásporas. Si bien las plantas exóticas no superan significativamente a las nativas en las relaciones fruto/flor y frutos consumidos/ frutos disponibles, las primeras tienen ventajas en la cantidad de semillas que incorporan al medio por unidad $\left(\mathrm{m}^{2}\right)$ de superficie de copa (ca. $1700 \mathrm{vs.} 800$ en la nativa que más dispersa), y en su germinación abundante (Ligustrum) o en la habilidad competitiva de sus plántulas (Rubus). El éxito de las malezas se explicaría en gran parte por su agresividad intrínseca y por la situación de marginalidad ecológica de esta comunidad selvática, ya que muchas especies se encuentran en el extremo meridional de su distribución.
\end{abstract}

Palabras clave: malezas leñosas, plantas exóticas, aves frugívoras, frugivoría, dispersión de semillas.

\begin{abstract}
Reproductive phenology, diaspore production, diaspore removal, and seed-dispersal by birds of five indigenous and two alien (Rubus ulmifolius and Ligustrum lucidum) woody species were studied in a riparian forest relict located in central Argentina (Punta Lara, Buenos Aires Province). Seed germination, recruitment, and seedling traits of these plants were also determined. During the last half century the weeds heavily invaded the forest, presently constituting a serious threat to the survival of this natural community. In the area the fruit-eating bird assemblage is reduced to only seven resident species and a migratory one, and some native plant species have reproductive constraints imposed by scarce fruit production or limited seed dispersal. Although the fruit : flower ratio and the ratio removed fruits : available fruits did not significantly differ between native and alien plants, the contribution of exotic plants to the seed pool were higher than that of indigenous plants (ca. 1700 seeds dispersed per canopy unit $-\mathrm{m}^{2}$ - vs. 800 seeds for the best dispersed native species). Aliens have advantages due to the seed germination rate (Ligustrum) or seedling competitive ability (Rubus) too. Many native species reach their southernmost distribution at the Punta Lara forest; the latter's current depauperate condition is probably mainly explained by the weeds' intrinsic invasiveness coupled with suboptimal habitat location for native plants.
\end{abstract}

Key words: woody weeds, exotic plants, fruit-eating birds, frugivory, seed dispersal.

\section{INTRODUCCION}

En muchas partes del mundo las plantas introducidas, principalmente leñosas, invaden las comunidades naturales y constituyen una seria amenaza para su supervivencia (Heywood 1989, Timmins \& Williams 1991). Aunque es difícil predecir si una especie será invasora (Noble 1989), existe una serie de caracteres favorables para que una planta se transforme en maleza (Noble 1989 y referencias en éste, Reichard \& Hamilton 1997). En el caso de las que tienen frutos carnosos la presencia de dispersores potenciales las convierte casi siempre en probables invasoras (Rejmánek \& Richardson 1996) y, por cierto, hay numerosas especies ornitócoras exóticas que se tornaron 
malezas (e.g. Knight 1986, Debussche \& Isenmann 1990 , White \& Stiles 1992 ). Ello ocurre porque las relaciones mutualistas entre las plantas y las aves frugívoras dispersoras de sus semillas son poco específicas (Herrera 1985, Jordano 1987 , Fuentes 1995) y, por lo tanto, las asociaciones funcionales entre los caracteres de los frutos y los principales dispersores no implican su coevolución como un proceso causal (Herrera 1985, 1995, Fischer \& Chapman 1993, Jordano 1995a).

En Argentina dos especies exóticas han dañado $y$ amenazan destruir un valioso relicto de selva ribereña que se encuentra en la provincia de Buenos Aires. Estas especies son Ligustrum lucidum Ait. (= ligustro; Oleaceae), originario de China y muy consumido por las aves de la zona (Montaldo 1993), y Rubus ulmifolius Schott (= zarzamora; Rosaceae), nativa de la región Mediterránea, donde es dispersada por aves frugívoras (Jordano 1982). El estrato arbóreo de la selva está invadido por L. lucidum (observación personal) e incluye unas 16 especies nativas, nueve de las que tienen síndrome de ornitocoria (cf. Van der Pijl 1982). Los claros de la selva fueron colonizados por $R$. ulmifolius (observación personal). Existen estudios descriptivos de la flora (Cabrera \& Dawson 1944) y avifauna (Klimaitis \& Moschione 1987) de esta selva ribereña, pero no hay trabajos completos sobre las interacciones entre ambas. Dicha información sólo está disponible para una de las malezas (Montaldo 1993).

En las plantas con flores la reproducción comprende desde la floración hasta el establecimiento de las plántulas, y el rendimiento reproductivo hace una contribución directa a la adecuación o ajuste ("fitness", Barret \& Eckert 1990), dando una idea del potencial invasor de una especie (Noble 1989). Por ello, el propósito de este trabajo fue estudiar comparativamente el éxito reproductivo de las principales especies de plantas ornitócoras de la selva ribereña de Punta Lara, ya que diferencias en el mismo contribuirían a explicar la situación de deterioro de la vegetación indígena.

\section{MATERIALES Y METODOS}

\section{Sitio del estudio y plantas estudiadas}

El estudio se hizo en la Reserva Natural de Punta Lara, que fue creada en 1958 para proteger una fítoasociación considerada como el relicto de selva subtropical más austral del continente (Cabrera 1960). Una descripción del clima, suelo y comunidades vegetales de la reserva fue hecha por Cabrera \& Dawson (1944). La selva ocupa aproximadamente 10 ha y se encuentra ubicada sobre la ribera del Río de La Plata $\left(37^{\circ} 47^{\prime} \mathrm{S}\right.$ y $58^{\circ}$ 1 ' W, ver Montaldo 1993). Este núcleo selvático constituye el extremo meridional de la selva Paranaense, que en forma de galerías sigue los cursos de los ríos Paraná y Uruguay (Cabrera 1976).

Se trabajó con las dos malezas ( $R$. ulmifolius y L. lucidum) y, por razones de factibilidad, el análisis de las especies nativas (seleccionadas dentro del grupo de las dispersadas por aves frugívoras) se limitó a las cinco más frecuentes en la selva: Ocotea acutifolia (Nees) Mez. (laurel; Lauraceae), Allophylus edulis (Camb.) Radlk. (chal chal; Sapindaceae), Blepharocalyx salicifolius (H.B.K.) Berg. (anacahuita; Myrtaceae), Rapanea laetevirens Mez. (canelón; Myrsinaceae), y Citharexylum montevidense (Spreng.) Mold. (espina de bañado; Verbenaceae). En adelante, y para simplificar, en el texto se utilizará solamente el nombre genérico. Con el objeto de facilitar la comparación, en las Tablas y Figuras se colocó a las especies siempre en el mismo orden, apareciendo las malezas en último término y señaladas con un asterisco.

Entre marzo de 1990 y marzo de1997 se visitó la reserva de Punta Lara en 29 oportunidades. Durante un período de dos años (1990-92) se realizaron observaciones regulares y continuas, quincenales o mensuales, dependiendo el intervalo entre visitas del estado fenológico de los vegetales estudiados.

\section{Características de las plantas}

Se cosecharon frutos maduros para caracterizar los frutos y semillas y hacer el análisis químico de la pulpa según las técnicas AOAC (1990). En Rubus se usó el fruto íntegro ante la imposibilidad de separar las pequeñas y numerosas semillas sin deteriorar significativamente la pulpa, y no se consiguieron suficientes frutos de Allophylus para hacer este análisis. Para la descripción de los frutos y semillas se utilizaron dos muestras semejantes de frutos. En una se tomaron los registros morfométricos, el peso fresco de la masa de frutos y de semillas, y el peso seco de las semillas (constancia de peso en estufa a $70^{\circ} \mathrm{C}$ ). En la otra se calculó el peso seco de la pulpa (por diferencia entre el de las semillas, de la muestra anterior, y el de los frutos enteros).

La fenología reproductiva (floración y fructificación) fue registrada siguiendo el sistema de Ledesma (1953). En cada oportunidad se registró el estado fenológico de individuos marcados (ver 
más adelante) y se hizo una inspección general de la población de cada especie. También se estimó la producción de frutos y semillas por unidad de superficie de planta. Para ello se extrapolaron recuentos hechos en inflorescencias individuales $\mathrm{y}$ recuentos del número de inflorescencias por $\mathrm{m}^{2}$ de copa. En cada individuo se etiquetaron inflorescencias que fueron seguidas hasta el momento en que finalizó la remoción de los frutos por las aves. Se contaron sucesivamente las flores formadas, los frutos maduros y los remanentes (= no consumidos). También se contaron los frutos dañados y, para estimar la caída de frutos, se embolsaron infrutescencias testigo (en malla plástica). La eficiencia reproductiva se midió por el cociente número de frutos maduros/ número de flores producidas (Montaldo 1993), y la de dispersión por el cociente número de frutos removidos por aves frugívoras dispersoras/ número de frutos ofrecidos. La última fue obtenida a partir de la eficiencia de remoción de frutos [porcentaje de frutos tomados por frugívoros relativo al total de frutos, $=100-\left(\sum \%\right.$ de frutos remanentes $+\%$ de caída de frutos)] (ver también Jordano 1995b), la que fue ponderada por un índice [coeficiente de dispersión, $=(100-\%$ de predación $) / 100]$ confeccionado con datos de censos en el campo de aves consumidoras. La cantidad de semillas dispersadas se estimó combinando datos de producción de frutos, número de semillas por fruto y eficiencia de dispersión.

Para conocer la germinación de semillas y el reclutamiento de plántulas, a partir de julio de 1991 se sembraron semillas en macetas (300 x $220 \times 60 \mathrm{~mm}$ ) mantenidas al aire libre y a media sombra, procurando reproducir las condiciones naturales de la selva. Se registró el momento de germinación y el número de semillas germinadas, y en enero de 1993 se cosecharon las plántulas sobrevivientes. Se midió la altura de las mismas, pesos fresco y seco (en estufa a $70^{\circ} \mathrm{C}$ ), y área foliar (con un medidor portátil Li-Cor 3000). Como en Rubus se observó escasa germinación, se repitió el experimento al año siguiente. Además, en esta segunda oportunidad, se enterraron semillas (tres grupos de 100, envueltos en malla plástica) para comprobar qué ocurría con las que no germinaban. Al año y a los dos años se investigó su viabilidad (test de Tetrazolio, Moore 1985) y poder germinativo (incubadora a $20-30^{\circ} \mathrm{C}, 15 \mathrm{y}$ $9 \mathrm{~h})$.

\section{Elenco consumidor}

La composición y fenología de la avifauna del área fue estudiada realizando un censo base, reco- rriendo en cada oportunidad en que se visitó la reserva dos transectas existentes en la selva (más detalles en Montaldo 1993). Para cada vegetal se estableció el elenco específico de consumidores (la nomenclatura sigue a Narosky \& Yzurieta 1987) y las proporciones de dispersores y depredadores de semillas, calculándose un valor único de este índice para todo el período controlado. Se hicieron censos por especie $\left(30^{\prime}\right.$ por censo, total $36 \mathrm{~h} 30^{\prime}$ ) y se incluyeron observaciones adicionales obtenidas mientras se realizaban otras tareas. Se supuso igual consumo por visita para todas las especies de aves, aunque en realidad el "valor cuantitativo" de cada una de ellas depende de su tasa de visita y del número de semillas removidas por vez (Herrera \& Jordano 1981, Jordano 1982),

El rol de las aves frugívoras como dispersoras o depredadoras de semillas (incluyendo en esta categoría a las que dejan caer la semilla debajo la planta madre, e.g. Jordano 1992) se investigó mediante consultas bibliográficas (e.g., Foster 1987, Levey 1987), observación directa en el campo, análisis de heces y regurgitados de individuos capturados con redes de niebla y, para cinco especies, se mantuvo en un aviario un ejemplar testigo. Estas aves fueron alimentadas con frutos de las plantas en estudio (total $36 \mathrm{~h}$ ). Las redes fueron colocadas en forma irregular a lo largo del estudio (12 días, total $180 \mathrm{~h}$ red) y los individuos capturados $(n=13)$ fueron retenidos temporalmente en una bolsa de nylon negro.

Para interpretar algunas de las interacciones planta-ave existentes en la selva de Punta Lara se caracterizó las aves, midiéndose la longitud del pico, el ancho de fauces entre comisuras (Wheelwright 1985a) y el peso corporal. Revisando la colección de pieles del Museo Argentino de Ciencias Naturales "Bernardino Rivadavia" se obtuvieron datos adicionales para las especies.

\section{Deterioro de la selva}

Por su significado biológico y ecológico interesa conocer la cantidad de semillas incorporadas al ecosistema (Noble 1989), variable que (entre otros factores, como por ejemplo el éxito de la polinización) depende del tamaño de los individuos de la especie y de su densidad en la comunidad (Mateucci \& Colma 1982). Como con excepción de Rubus (un arbusto) y Allophylus (un árbol menor), los restantes son árboles de la misma magnitud, la unidad de comparación tomada $\left(\mathrm{m}^{2}\right.$ de superficie de copa, ver Características de las plantas) da una idea aproximada de la producción y dispersión de diásporas por individuo. Para 
estimar la densidad poblacional, en la selva se trazaron al azar cinco parcelas de $5 \mathrm{~m}$ de lado y se censaron los ejemplares arbóreos en condiciones de fructificar. Adicionalmente se recorrió una transecta de ca. $300 \mathrm{~m}$ (ver Montaldo 1993) y se anotaron los individuos reproductivos situados a ambos lados de la misma. No se consideraron los claros de la selva porque, como se dijo, están ocupados por Rubus. Se comparó el resultado con el obtenido por Cabrera \& Dawson (1944), que hicieron un relevamiento semejante pero en cuatro parcelas de $10 \mathrm{~m}$ de lado.

\section{Análisis de datos}

Debido a su mayor potencia-eficiencia se prefirió la utilización de métodos estadísticos paramétricos. Cuando los datos estuvieron expresados en porcentaje antes del análisis se aplicó la transformación angular o arco-seno (Sokal \& Rohlf 1969). En el test de $X^{2}$ se usó la corrección de continuidad de Yates (Siegel 1975).

\section{RESULTADOS}

\section{Fenología de las plantas}

La oferta de frutos de las dos malezas consideradas no coincide con la de la mayoría de las especies nativas (Fig. 1). Mientras que éstas maduran sus frutos en otoño (salvo Allophylus, que ofrece sus frutos en primavera tardía), Rubus lo hace en pleno verano y Ligustrum en invierno y primavera, cuando existe baja disponibilidad de alimento en el ecosistema (observación personal). Los frutos de la última coexisten durante la estación fría con los de Citharexylum, que aunque maduran en otoño permanecen largo tiempo sobre las plantas.

\section{Caracteres de los frutos y semillas}

En tres de las especies el tamaño del fruto (Tabla 1) condiciona su elenco de dispersores. Ocotea y Rubus tienen frutos de diámetro semejante al ancho medio de las fauces de las aves frugívoras de Punta Lara (Tabla 3, $\bar{x}=12,2 \mathrm{~mm}$ ) (test $\mathrm{t}, \mathrm{t}_{26}$ $=1,38$ para Ocotea y t ${ }_{26}=0,11$ para Rubus, $\mathrm{P}>$ 0,05). Los de Citharexylum, si bien son algo menores $\left(\mathrm{t}_{26}=3,32, \mathrm{P}<0,01\right)$, su pulpa firme $\mathrm{y}$ adherida al endocarpo contribuye a dificultar la manipulación del fruto (Levey 1987) por los frugívoros pequeños, que no pueden aplastarlo o despedazarlo para ingerirlo (e.g. Stephanophorus diadematus, observación personal). Dos especies nativas (Ocotea y Rapanea) tienen altos valores de lípidos en el pericarpo (Tabla 2), pero la mayor parte del fruto está ocupado por la semilla. El bajo contenido proteico de los frutos estudiados, su elevado contenido de agua y el porcentaje de hidratos de carbonos estructurales (fibra) no difieren de lo conocido para las especies ornitócoras

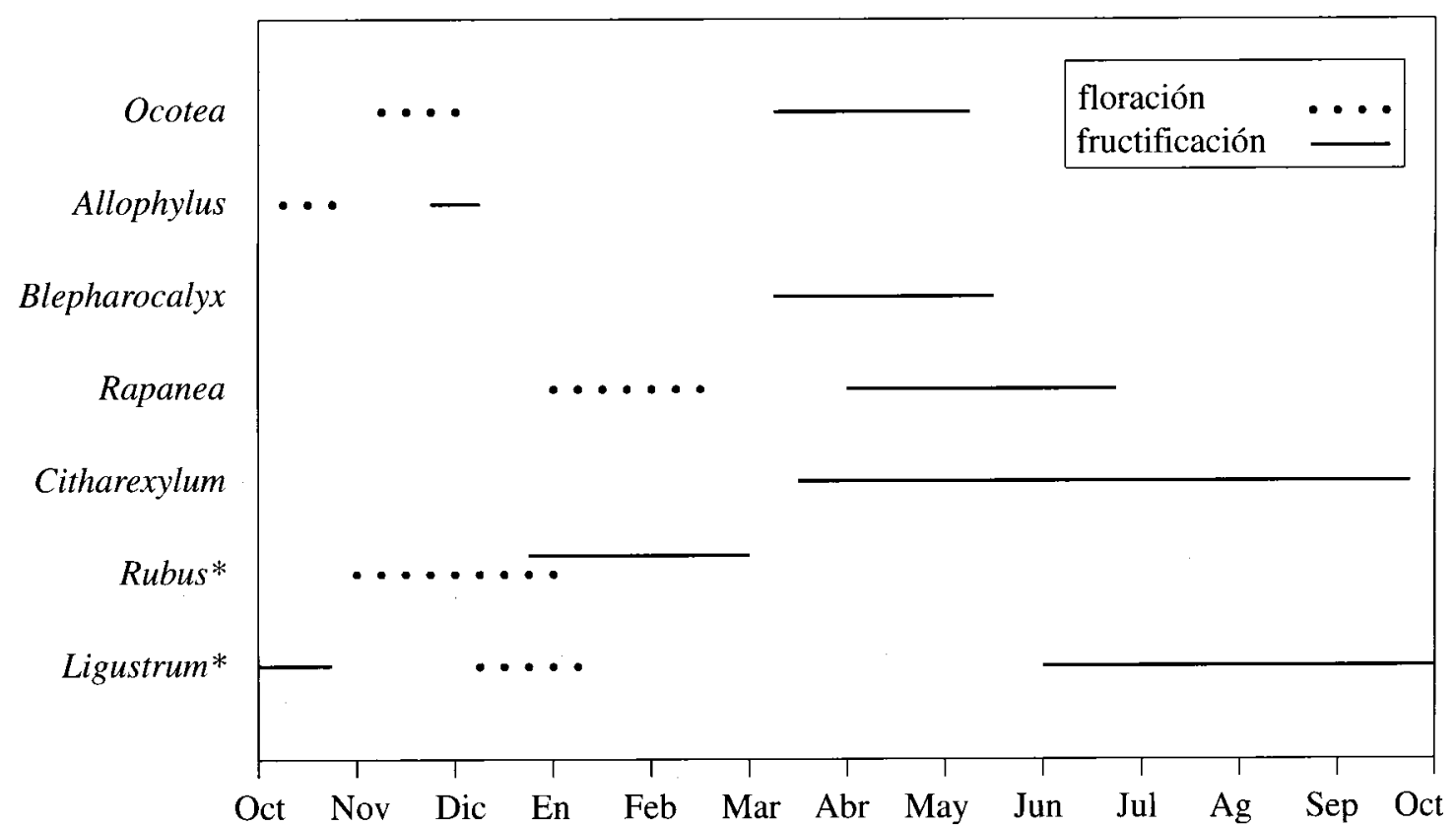

Fig. 1. Fenología reproductiva de las plantas ornitócoras de la selva de Punta Lara durante los años 1990-1992. Línea de puntos: floración, línea entera: frutos maduros.

Reproductive phenology of the bird-dispersed plant species at the Punta Lara forest, 1990-1992. Dotted line: flowering, solid line: ripe fruits. 
TABLA 1

Caracteres de los frutos y semillas de las especies ornitócoras de la selva de Punta Lara

Fruits and seeds characteristics of the bird-dispersed plant species at the Punta Lara forest

\begin{tabular}{|c|c|c|c|c|c|c|c|}
\hline Especie & $\begin{array}{c}\text { Tipo } \\
\text { de fruto }\end{array}$ & $\begin{array}{l}\text { Color }{ }^{1} \\
\text { del fruto }\end{array}$ & $\begin{array}{l}\text { Diámetro } \\
\text { del fruto } \\
(\mathrm{mm})\end{array}$ & $\begin{array}{c}\text { Tipo } \\
\text { de pulpa }\end{array}$ & $\begin{array}{l}\text { Semillas } \\
\text { por fruto }\end{array}$ & $\begin{array}{c}\text { Diámetro } \\
\text { de la semilla } \\
(\mathrm{mm})\end{array}$ & $\mathrm{P} / \mathrm{S}^{2}$ \\
\hline Ocotea acutifolia & baya & verde & $13,6 \pm 0,7$ & firme & 1 & $10,7 \pm 0,6$ & 0,9 \\
\hline Allophylus edulis & drupa & rojo & $6,8 \pm 0,2$ & blanda & 1 & $4,6 \pm 0,1$ & 3,4 \\
\hline Blepharocalyx salicifolius & baya & rojo & $6,9 \pm 0,2$ & blanda & $\begin{array}{c}1,3 \pm 0,2 \\
(1-5)\end{array}$ & $2,9 \pm 0,1$ & 6,3 \\
\hline Rapanea laetevirens & drupa & negro & $5,3 \pm 0,1$ & firme & 1 & $4,1 \pm 0,1$ & 1,2 \\
\hline Citharexylum montevidense & drupa & rojo & $9,6 \pm 0,2$ & firme & 2 & $4,6 \pm 0,0$ & 4,3 \\
\hline Rubus ulmifolius* & drupa & negro & $12,3 \pm 0,6$ & blanda & $\begin{array}{c}20,8 \pm 2,6 \\
(1-38)\end{array}$ & $2,2 \pm 0,0$ & 10,1 \\
\hline Ligustrum lucidum* & drupa & negro & $5,9 \pm 0,1$ & blanda & $\begin{array}{c}1,2 \pm 0,1 \\
(1-2)\end{array}$ & $3,8 \pm 0,1$ & 1,8 \\
\hline
\end{tabular}

Valores $=$ media \pm error estándar, y rango (entre paréntesis). $\mathrm{N}=20$ frutos/ semillas por especie.

${ }^{1}$ En el color negro se incluyen el púrpura y el azul oscuro (cf. Wheelwright \& Janson, 1985).

${ }^{2}$ Peso fresco del pericarpo/ peso fresco de las semillas.

en otras partes (Herrera 1987). Los altos valores de proteína y fibra de $R$ ubus se deberían a que en el análisis de esta especie se incluyeron las semillas (ver Métodos), por lo que los hidratos de carbono no estructurales estarían subestimados (ver Snow 1971, para $R$. idaeus, y Herrera 1987, para Rosaceae en general). Los frutos de una de las exóticas (Ligustrum) tienen los porcentajes más bajos de nutrientes aprovechables para las aves (proteína, lípidos, hidratos de carbono no estructurales) (Tabla 2) y también una relación pulpa/ semilla relativamente baja (Tabla 1). En el caso de la otra (Rubus), que posee el índice más favorable, si se considera el receptáculo (que es ingerido por las aves que tragan el fruto íntegro), esta relación desciende a 5,4. En cuanto al número de semillas por fruto, esta maleza posee una cantidad muy superior a las demás especies (Tabla 1).

TABLA 2

Composición porcentual de los frutos de las especies ornitócoras de la selva de Punta Lara Percentage composition of fruits of the bird-dispersed plant species at the Punta Lara forest

\begin{tabular}{lcccccc}
\hline Especie & Agua & $\begin{array}{c}\text { Proteina } \\
\text { cruda }\end{array}$ & $\begin{array}{c}\text { Lípidos } \\
\text { totales }\end{array}$ & Fibra cruda & $\begin{array}{c}\text { Hidratos de } \\
\text { carbono no } \\
\text { estructurales }\end{array}$ & Cenizas \\
\hline Ocotea acutifolia & $73,2 \pm 4,6$ & $6,2 \pm 0,6$ & $35,0 \pm 1,5$ & $30,2 \pm 2,6$ & $11,1 \pm 0,6$ & $2,9 \pm 0,2$ \\
Allophylus edulis & $77,1 \pm 7,5$ & $\mathrm{~s} / \mathrm{d}$ & $\mathrm{s} / \mathrm{d}$ & $\mathrm{s} / \mathrm{d}$ & $\mathrm{s} / \mathrm{d}$ & $\mathrm{s} / \mathrm{d}$ \\
Blepharocalyx salicifolius & $80,7 \pm 4,6$ & $3,9 \pm 0,6$ & $10,5 \pm 0,2$ & $5,0 \pm 0,3$ & $42,0 \pm 2,9$ & $4,5 \pm 0,3$ \\
Rapanea laetevirens & $64,6 \pm 6,3$ & $4,2 \pm 0,2$ & $36,2 \pm 2,2$ & $11,6 \pm 0,6$ & $27,5 \pm 3,0$ & $6,3 \pm 0,6$ \\
Citharexylum montevidense & $77,1 \pm 7,8$ & $3,8 \pm 0,3$ & $2,8 \pm 0,2$ & $5,9 \pm 0,5$ & $48,9 \pm 2,5$ & $4,5 \pm 0,4$ \\
Rubus ${ }^{1}$ ulmifolius* & $89,1 \pm 3,2$ & $12,1 \pm 0,4$ & $1,1 \pm 0,2$ & $41,6 \pm 3,7$ & $35,1 \pm 3,8$ & $5,1 \pm 0,5$ \\
Ligustrum lucidum * & $76,8 \pm 2,9$ & $4,2 \pm 0,5$ & $3,8 \pm 0,6$ & $29,9 \pm 3,3$ & $19,0 \pm 2,2$ & $6,9 \pm 1,6$ \\
\hline
\end{tabular}

Con excepción del contenido de humedad, todos los porcentajes son sobre base seca. $\mathrm{S} / \mathrm{d}=$ sin datos. Valores $=$ media \pm error estándar. Se hicieron tres determinaciones por especie.

${ }^{1}$ Incluye las semillas (ver texto). 
Interacciones planta-ave

La avifauna de la selva de Punta Lara está integrada por unas 37 especies de aves, y la mitad de ellas tiene hábitos frugívoros (Phelps \& Meyer de Schauensee 1979, Sick 1986, Foster 1987). En la Fig. 2 se incluyeron sólo las que se consideraron de alguna importancia ecológica para los vegetales, sea por tener una población abundante o por su elevado consumo de frutos. Ocho especies son las principales consumidoras de frutos de la comunidad, de las que sólo una es migratoria (Tabla 3). El resto de las aves frugívoras que arriban al área en primavera-verano (ver Montaldo 1993) no consumen frutos en ese período o lo hacen en baja medida (observación personal).

Las dos especies de Tyrannidae dispersan las semillas de todas las plantas con que interactúan, las de Columbidae son depredadoras y las de Turdidae y Thraupidae desempeñan ambos roles, según el vegetal del que se alimenten. En este lugar los principales dispersores son los zorzales (Turdidae), mientras que una paloma (Columba picazuro) es una importante destructora de semillas. En el caso de Citharexylum se debe señalar, no obstante, que algunos de sus carozos logran atravesar el tracto digestivo de $C$. picazuro y al ser defecados contienen semillas viables. De 50 carozos ingeridos por un individuo cautivo de esta paloma, $8(=16 \%)$ aparecieron enteros o poco dañados en las deyecciones, y algunos de ellos originaron plántulas normales ( $8 \%$ ). Con excepción de Citharexylum, el resto de las plantas interactúa principalmente con un dispersor (Fig. 2). Si bien el tamaño de los frutos de Citharexylum permite que sean ingeridos fácil- mente por Turdus spp. (test $\mathrm{t}, \mathrm{t}_{24}=8,85$ para $T$. rufiventris, $\mathrm{y}_{24}=9,19$ para $T$. amaurochalinus, $\mathrm{P}<$ 0,01 ), al parecer estas aves son poco atraídas por dicha especie (ver más adelante, Discusión).

\section{Producción y dispersión de diásporas}

Durante el período 1990-92 la especie más florífera fue Ligustrum, que produjo en cada ciclo unas 33000 flores por $\mathrm{m}^{2}$ de copa (Tabla 4), mientras que la otra maleza (Rubus) fue la que formó menos flores (con poco más de 200). Si bien la producción de flores de Ligustrum puede ser en algún año de poco más de la mitad de la cifra mencionada (Montaldo 1993), supera claramente a las plantas nativas, que en ningún caso alcanzaron las 2000 flores por $\mathrm{m}^{2}$ de copa.

En cuanto a los frutos formados, la eficiencia reproductiva media varió con la especie (Fig. 3) (ANOVA de dos factores, $\mathrm{F}_{6-28}=32,08, \mathrm{P}<0,01$ ) $y$ no con el año $\left(F_{1-28}=0,32, P>0,05\right)$, y la interacción especie por año no resultó significativa $\left(\mathrm{F}_{6-28}=1,80, \mathrm{P}>0,05\right)$. Rubus y dos especies nativas (Rapanea y Citharexylum) produjeron aproximadamente un fruto cada dos flores, mientras que en Ligustrum, una de cada diez flores llegó al estado de fruto maduro (a veces dos, Montaldo 1993). Allophylus y Blepharocalyx fueron aún menos eficientes que Ligustrum y tuvieron una proporción muy baja de frutos logrados ( $1-1,5 \%)$, mientras que Ocotea tuvo un desempeno intermedio entre estas especies.

La proporción de frutos maduros consumidos por aves fue similar en ambos años (ANOVA de

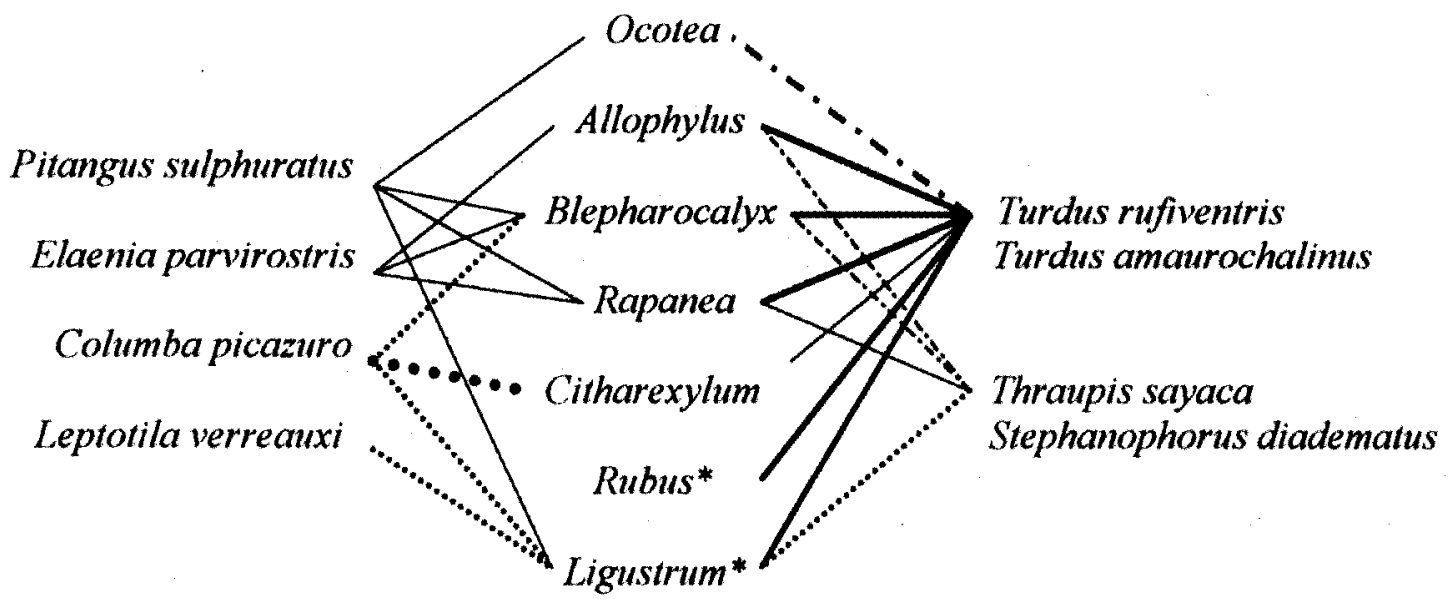

Fig. 2. Principales interacciones planta-ave en la selva de Punta Lara. Línea gruesa: consumidor primario (representa el 50\% o más del elenco específico); fina: consumidor secundario (menos del $50 \%$ ). Línea entera: dispersor; de puntos: predador; de rayas y puntos: desempeña ambos roles.

Main plant-bird interactions at the Punta Lara forest. Heavy line: major consumer (represents $50 \%$ or more of the specific assemblage); light line: secondary consumer (fewer than 50\%). Solid line: disperser; dotted line: predator; dashed and dotted line: disperser and predator. 
TABLA 3

Caracteres y estatus de las principales especies de aves frugívoras en la selva de Punta Lara

Morphological characteristics and residency status of the main fruit-eating birds at the Punta Lara forest

\begin{tabular}{|c|c|c|c|c|c|}
\hline Especie & Familia & $\begin{array}{l}\text { Ancho de fauces } \\
(\mathrm{mm})\end{array}$ & $\begin{array}{l}\text { Longitud } \\
\text { del pico }(\mathrm{mm})\end{array}$ & $\begin{array}{l}\text { Peso } \\
(\mathrm{g})\end{array}$ & Estatus \\
\hline Leptotila verreauxi & Columbidae & $9,2 \pm 0,8$ & $19,6 \pm 0,9$ & $S / d$ & $\begin{array}{l}\text { residente } \\
\text { común }\end{array}$ \\
\hline Columba picazuro 1 & Columbidae & $14,2 \pm 1,6$ & $18,6 \pm 1,7$ & $269,0 \pm 57,8(3)$ & $\begin{array}{l}\text { residente } \\
\text { común }\end{array}$ \\
\hline Pitangus sulphuratus 1 & Tyrannidae & $17,7 \pm 2,0$ & $31,0 \pm 1,4$ & $66,5 \pm 2,8(2)$ & $\begin{array}{l}\text { residente } \\
\text { común }\end{array}$ \\
\hline Elaenia parvirostris & Tyrannidae & $7,7 \pm 1,0$ & $9,7 \pm 0,5$ & $17,0(1)$ & $\begin{array}{c}\text { migratoria } \\
\text { abundante }^{2}\end{array}$ \\
\hline Turdus rufiventris 1 & Turdidae & $14,2 \pm 1,8$ & $20,8 \pm 1,0$ & $74,4 \pm 5,9 \cdot(6)$ & $\begin{array}{l}\text { residente } \\
\text { abundante }\end{array}$ \\
\hline Turdus amaurochalinus & Turdidae & $13,6 \pm 1,3$ & $19,2 \pm 0,7$ & $65,3 \pm 1,2(3)$ & $\begin{array}{l}\text { residente } \\
\text { común }\end{array}$ \\
\hline Thraupis sayaca ${ }^{1}$ & Thraupidae & $10,3 \pm 0,7$ & $13,8 \pm 1,3$ & $32,2 \pm 5,6$ & $\begin{array}{l}\text { residente } \\
\text { escaso }\end{array}$ \\
\hline Stephanophorus diadematus 1 & Thraupidae & $10,7 \pm 1,0$ & $11,2 \pm 1,0$ & $37,6 \pm 1,3(2)$ & $\begin{array}{l}\text { residente } \\
\text { escaso }\end{array}$ \\
\hline
\end{tabular}

Valores = media \pm desviación estándar. $\mathrm{N}=6$ individuos por especie, excepto para el peso (entre paréntesis). S/d = sin datos.

${ }^{1}$ Especies en las que se mantuvo un ejemplar cautivo.

${ }^{2}$ Visitante primavero-estival.

dos factores, $\left.F_{1-28}=0,21, P>0,05\right)$ y dependió de cada especie $\left(\mathrm{F}_{6-28}=41,03, \mathrm{P}<0,01\right)$, no verificándose interacción entre año y especie $\left(\mathrm{F}_{6-28}=\right.$ $0,43, P>0,05)$. En las plantas nativas la remoción de frutos fue superior que en las exóticas (Fig. 4).

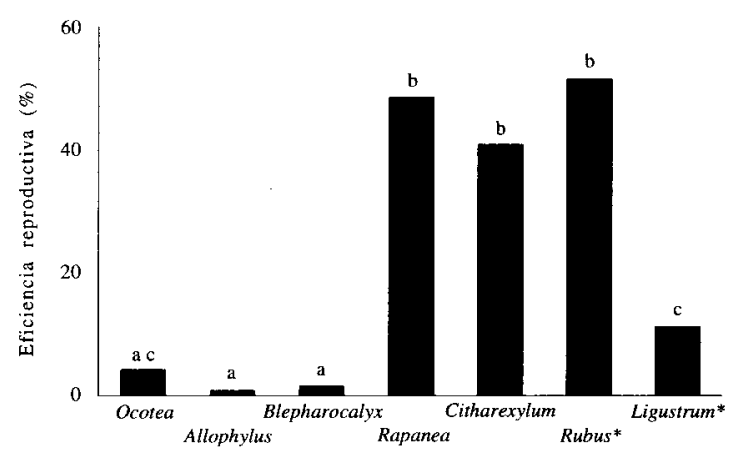

Fig. 3. Eficiencia reproductiva (frutos maduros/ flores formadas) de las especies ornitócoras de la selva de Punta Lara en el período 1990-1992.

Columnas con la misma letra no difieren entre sí (test de Tukey, $\mathrm{P}>0,05$ ).

Reproductive efficiency (ripe fruits : flowers ratio) of the bird-dispersed plant species at the Punta Lara forest, 19901992. Bars with the same letter are not significantly different (Tukey's studentized range test, $\mathrm{P}>0,05$ ).

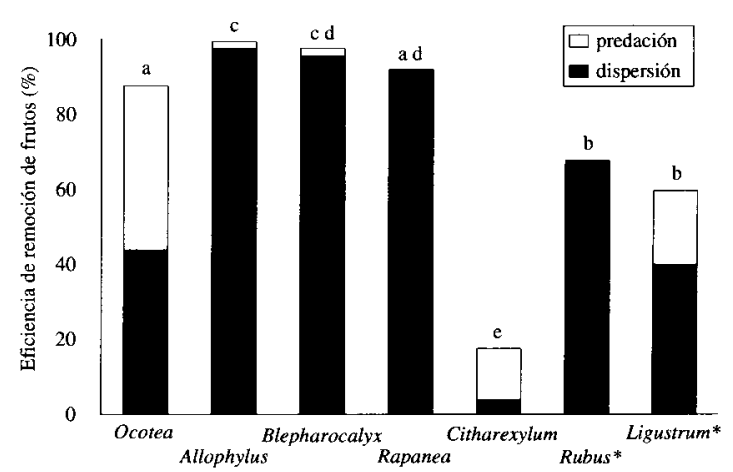

Fig. 4. Eficiencia de remoción de frutos de las especies ornitócoras de la selva de Punta Lara en el período 1990-92. Coeficiente de dispersión para Ocotea $=0,50 ;$ Rubus y Rapanea $=1 ;$ Allophylus y Blepharocalyx $=0,98 ;$ Ligustrum $=0,67$;

Citharexylum $=0,22$. Columnas con la misma letra no difieren entre sí (test de Tukey, $\mathrm{P}>0,01$ ).

Fruit removal efficiency of the bird-dispersed plant species at the Punta Lara forest, 1990-1992. Open bar: fruit predation, solid bar: fruit dispersal. Dispersal rate for Ocotea $=0,50 ;$ Rubus and Rapanea $=1 ;$ Allophylus and Blepharocalyx $=0,98 ;$ Ligustrum $=0,67 ;$ Citharexylum $=$ 0,22 . Bars with the same letter are not significantly different (Tukey's studentized range test, $P>0,01$ ). 
La excepción fue Citharexylum, que tuvo la menor tasa de remoción y la mayoría de los frutos consumidos fueron depredados. También se detectó una fuerte pérdida de frutos en otra especie nativa (Ocotea), donde sólo la mitad de los removidos son efectivamente dispersados (Fig. 4). En Ocotea gran parte de los frutos cayó bajo la planta madre porque su tamaño no difiere mayormente del de las fauces de Turdus rufiventris (lo que dificulta la ingestión; test $\left.\mathrm{t}, \mathrm{t}_{24}=0,62, \mathrm{P}>0,05\right)$, que es su principal consumidor en Punta Lara. Además estos frutos son ofrecidos en el extremo de ramas delgadas, por lo que dichas aves deben tomarlos principalmente en vuelo. A pesar de que muchas de sus flores se pierden, Ligustrum aventaja ampliamente al resto de las especies en la cantidad de frutos ofrecidos y en la de semillas listas para ser dispersadas (Fig. 5). Rubus produce relativamente pocos frutos, pero éstos contienen un número de semillas muy superior al de las nativas. Las plantas exóticas logran su mayor producción de semillas a través de estrategias diferentes: muchos frutos pauciseminados en Ligustrum, y pocos frutos polispermos en Rubus.

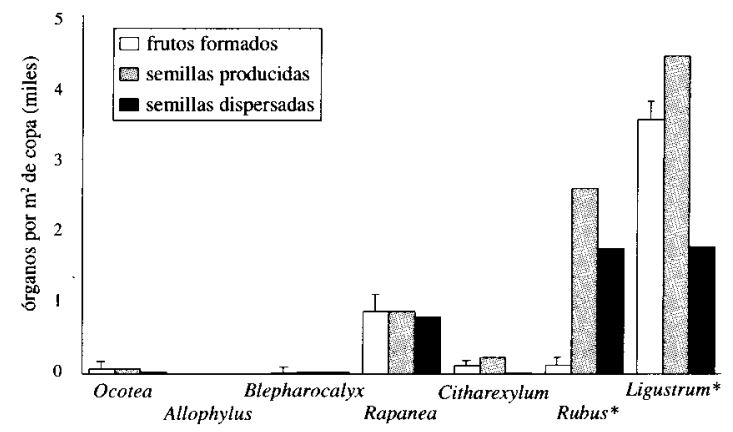

Fig. 5. Producción anual de diásporas y dispersión de semillas por unidad de superficie verde $\left(\mathrm{m}^{2}\right)$ de las especies ornitócoras de la selva de Punta Lara en el período 1990-1992. La línea representa el error estándar. Semillas producidas (frutos formados $\mathrm{x}$ semillas por fruto, Tabla 1 ); semillas dispersadas (semillas producidas $\mathrm{x}$ eficiencia de dispersión, Fig. 4).

Annual diaspore production and seed dispersal per canopy unit $\left(\mathrm{m}^{2}\right)$ of the bird-dispersed plant species at the Punta Lara forest, 1990-1992. Open bar: fruit production (line is the standar error); dotted bar: seed production (fruits produced $x$ seeds per fruit, Table 1); solid bar: seed dispersal (seeds produced $x$ fruit dispersal efficiency, Fig. 4).

TABLA 4

Producción de estructuras reproductivas de las especies ornitócoras en la selva de Punta Lara durante los ciclos 1990-91 (línea superior) y 1991-92 (línea inferior)

Production of reproductive structures in the the bird-dispersed plant species at the Punta Lara forest, in 199091(upper line) and 1991-92 (lower line)

\begin{tabular}{|c|c|c|c|c|}
\hline Especie & $\begin{array}{l}\text { Flores por } \\
\text { inflorescencia }\end{array}$ & $\begin{array}{l}\text { Frutos por } \\
\text { infrutescencia }\end{array}$ & $\begin{array}{l}\text { Inflorescencias } \\
\text { por } \mathrm{m}^{2} \text { de copa }\end{array}$ & $\begin{array}{r}\text { Flores por } \mathrm{m}^{2} \mathrm{de} \\
\text { copa }(\text { col. } 2 \times \text { col. } 4)\end{array}$ \\
\hline \multirow[t]{2}{*}{ Ocotea acutifolia } & $92,2 \pm 15,8$ & $2,6 \pm 2,5$ & $25,4 \pm 15,8$ & 2341,9 \\
\hline & $90,8 \pm 8,9$ & $5,1 \pm 1,3$ & $17,7 \pm 8,5$ & 1607,2 \\
\hline \multirow[t]{2}{*}{ Allophylus edulis } & $8,4 \pm 2,7$ & $0,1 \pm 0,1$ & $205,4 \pm 157,6$ & 1725,4 \\
\hline & $9,3 \pm 3,5$ & $0,0 \pm 0,0$ & $133,1 \pm 98,1$ & 1237,8 \\
\hline \multirow[t]{2}{*}{ Blepharocalyx salicifolius } & $4,7 \pm 1,3$ & $0,1 \pm 0,1$ & $267,5 \pm 127,4$ & 1257,3 \\
\hline & $4,7 \pm 1,0$ & $0,0 \pm 0,0$ & $417,3 \pm 159,6$ & 1961,3 \\
\hline \multirow[t]{2}{*}{ Rapanea laetevirens } & $6,0 \pm 1,2$ & $3,4 \pm 0,6$ & $331,5 \pm 39,5$ & 1989,0 \\
\hline & $6,9 \pm 0,6$ & $2,8 \pm 1,3$ & $246,1 \pm 101,1$ & 1698,1 \\
\hline \multirow[t]{2}{*}{ Citharexylum montevidense } & $31,5 \pm 7,4$ & $8,7 \pm 6,9$ & $22,9 \pm 13,8$ & 721,4 \\
\hline & $19,8 \pm 1,1$ & $10,2 \pm 0,5$ & $9,0 \pm 4,2$ & 178,2 \\
\hline \multirow[t]{2}{*}{ Rubus ulmifolius* } & $22,2 \pm 12,0$ & $12,6 \pm 6,4$ & $10,2 \pm 2,0$ & 226,4 \\
\hline & $25,6 \pm 3,0$ & $15,3 \pm 10,6$ & $8,6 \pm 1,1$ & 220,2 \\
\hline \multirow[t]{2}{*}{ Ligustrum lucidum* } & $1763,1 \pm 116,6$ & $206,1 \pm 23,8$ & $15,1 \pm 2,5$ & 26622,8 \\
\hline & $1500,8 \pm 852,5$ & $127,3 \pm 24,2$ & $26,5 \pm 7,7$ & 39771,2 \\
\hline
\end{tabular}

Valores = media \pm desviación estándar. $\mathrm{N}=3$ individuos por especie/ 15 inflorescencias o grupos de inflorescencias por individuo/ 4 áreas por individuo controlado.

Las diferencias entre ciclos reproductivos no fueron significativas (test $t$ de Student, $P>0,05$ ). 


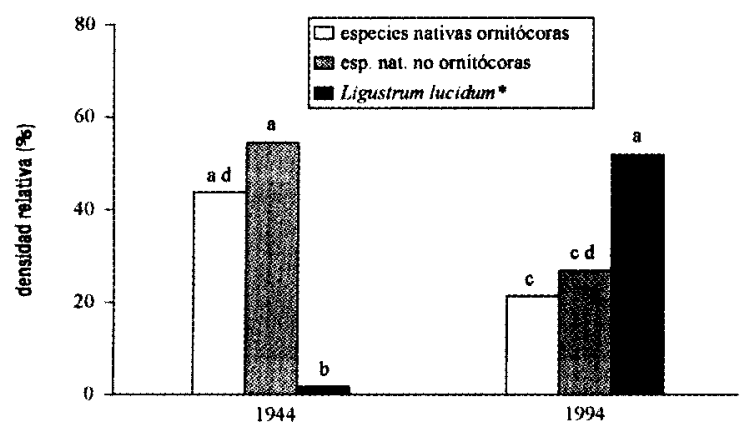

Fig. 6. Variación de la densidad poblacional en la selva de Punta Lara en el intervalo1944-1994. Columnas con la misma letra no difieren entre sí (test de Tukey, $\mathrm{P}>0,05$ ).

Population density variation at the Punta Lara forest between 1944 and 1994. Open bar: native bird-dispersed plant species; dotted bar: native non-ornithochorous plant species; solid bar: Ligustrum lucidum. Bars with the same letter are not significantly different (Tukey's studentized range test, $\mathrm{P}>0,05$ ).

También las malezas son las plantas que más semillas dispersan por unidad de superficie de planta; sin embargo, una nativa (Rapanea) logra dispersar casi ochocientas semillas por unidad de referencia (Fig. 5).

\section{Densidad poblacional y deterioro de la selva}

Considerando con fines comparativos una superficie productora de diásporas similar en todas las especies estudiadas, en Punta Lara las malezas (Ligustrum y Rubus) producirían el $77 \%$ de los frutos ofrecidos a las aves durante el año, el $85 \%$ de las semillas en condiciones de ser dispersadas, y el $80 \%$ de las semillas que se incorporan al ecosistema. Pero la realidad de la selva de Punta Lara es más grave que esta situación hipotética, ya que (aún prescindiendo de $R u b u s$ ) el número de individuos de Ligustrum reproductivamente activos supera al del conjunto de las especies nativas ornitócoras (no sólo las consideradas en este estudio), y también al de las que no lo son (ANOVA, $F_{5-21}=9,05, P<0,01$ ) (Fig. 6). En el presente la población de individuos productores de diásporas de la exótica es equivalente a la de todas las especies nativas $(\overline{\mathrm{x}}=51,8$ vs. $48,2 \%$, Test $\left.\mathrm{t}, \mathrm{t}_{8}=0,38, \mathrm{P}>0,05\right)$. La Fig. 6 muestra el resultado medio de los censos realizados en las parcelas (donde se hallaron 54 ejemplares reproductivos), que fue similar al obtenido en la transecta de $300 \mathrm{~m}$ (de 123 individuos registrados, el 56,9\% correspondía a Ligustrum, 20,3\% a las especies nativas ornitócoras y $22,8 \%$ a las no ornitócoras). La comparación con los datos de Cabrera \& Dawson (1944) permite ver que, medio siglo después, la composición florística y estructural de la selva ha cambiado radicalmente (Fig. 6), modificándose en forma importante su fisonomía (ver también Dascanio \& Ricci 1988).

\section{Germinación y supervivencia de plántulas}

En las condiciones del ensayo Ligustrum fue la especie cuyas semillas tuvieron mayor poder germinativo y también la de mejor relación plántulas obtenidas/ semillas dispersadas, aunque la diferencia con una especie nativa (Blepharocalyx) no fue significativa (Tabla 5).

TABLA 5

Germinación de semillas y supervivencia de plántulas de las especies ornitócoras de la selva de Punta Lara

Seed germination and seedling survival of the the bird-dispersed plant species of the Punta Lara forest

\begin{tabular}{|c|c|c|c|}
\hline Especie & Semillas sembradas & Semillas germinadas & Plántulas establecidas \\
\hline Ocotea acutifolia & 100 & $65(65)^{a}$ & $65(65)^{a}$ \\
\hline Allophylus edulis & 25 & $14(56)^{a}$ & $10(40)^{b}$ \\
\hline Blepharocalyx salicifolius & 100 & $86(86) b d$ & $78(78)^{\text {ad }}$ \\
\hline Rapanea laetevirens & 100 & $84(84)^{b}$ & $70(70)^{a}$ \\
\hline Citharexylum montevidense & 100 & $53(53)$ a & $36(36)^{b}$ \\
\hline Rubus ulmifolius* & 100 & $12(12)^{c}$ & $12(12)^{c}$ \\
\hline Ligustrum lucidum* & 100 & $95(95)^{d}$ & $87(87)^{d}$ \\
\hline
\end{tabular}

$\%$ entre paréntesis. Valores con la misma letra no difieren entre sí (test de $\mathrm{X}^{2}, \mathrm{P}>0,05$ ). 
Caracteres de las plántulas de las especies ornitócoras de la selva de Punta Lara

Seedling features of the bird-dispersed plant species of the Punta Lara fores

\begin{tabular}{|c|c|c|c|c|c|c|}
\hline \multirow[t]{2}{*}{ Especie } & \multirow[t]{2}{*}{$\begin{array}{c}\text { Altura } 1 \\
(\mathrm{~mm})\end{array}$} & \multirow[t]{2}{*}{$\begin{array}{l}\text { Area foliar } \\
\qquad\left(\mathrm{mm}^{2}\right)\end{array}$} & \multirow[t]{2}{*}{ Peso fresco $(\mathrm{g})$} & \multirow[t]{2}{*}{$\begin{array}{c}\text { Peso } \\
\operatorname{seco}(\mathrm{g})\end{array}$} & \multicolumn{2}{|c|}{$\begin{array}{l}\text { Tiempo } \\
\text { (meses) }\end{array}$} \\
\hline & & & & & $S-G$ & S-C ${ }^{2}$ \\
\hline Ocotea acutifolia & $348 \pm 101 \mathrm{a}$ & $10120 \pm 4010^{a}$ & $2,6 \pm 0,9$ a & 0,6 & 3,5 & 9,0 \\
\hline Allophylus edulis & $129 \pm 37 b$ & $7970 \pm 5290$ ac & $1,1 \pm 0,7^{b}$ & 0,3 & 0,5 & 12,5 \\
\hline Blepharocalx salicifolius & $120 \pm 50^{b}$ & $2180 \pm 1430^{b}$ & $0,3 \pm 0,2^{\mathrm{c}}$ & 0,1 & 2,0 & 9,0 \\
\hline Rapanea laetevirens & $132 \pm 31 b$ & $4200 \pm 2970^{b}$ & $1,2 \pm 0,8^{b}$ & 0,2 & 3,5 & 17,5 \\
\hline Citharexylum montevidense & $149 \pm 65 b c$ & $5240 \pm 4480 \mathrm{bc}$ & $1,2 \pm 1,1 b$ & 0,3 & 1,5 & 17,5 \\
\hline Rubus ulmifolius* & $174 \pm 66$ bc & $9980 \pm 4700 \mathrm{a}$ & $1,4 \pm 0,7 b$ & 0,4 & 8,0 & 11,5 \\
\hline Ligustrum lucidum* & $201 \pm 46^{c}$ & $4560 \pm 2940 \mathrm{bc}$ & $1,1 \pm 0,7 \mathrm{~b}$ & 0,3 & 1,5 & 17,5 \\
\hline
\end{tabular}

Valores = media \pm desviación estándar (altura, área foliar y peso fresco), media (peso seco). $\mathrm{N}=10$ plántulas por especie. Valores con la misma letra no difieren entre sí (test de Tukey, para altura $\mathrm{P}>0,01$; área foliar y peso fresco $\mathrm{P}>0,05$ ).

${ }^{1}$ En Rubus largo del tallo principal (es decumbente).

${ }^{2}$ Siembra-germinación (emergencia de las primeras plántulas) y siembra-cosecha.

Sin embargo, los períodos de control para ambas especies fueron diferentes (Tabla 6), por lo que es probable que en igual tiempo Ligustrum reclute más plántulas que Blepharocalyx. Las semillas de Rubus fueron las que más tardaron en germinar (Tabla 6) y las que lo hicieron en menor medida (Tabla 5), registrándose germinación nula cuando se repitió el experimento (ver Métodos). No obstante, en este último caso el $52 \%$ de las semillas permanecían viables al año de sembradas y el $34 \%$ germinó cuando se las sometió a temperaturas alternadas. Un año después, el $29 \%$ mantenía ambas propiedades. Las pocas plántulas de Rubus que nacieron en el primer ensayo progresaron vigorosamente, y en un lapso comparativamente corto (tres meses y medio, Tabla 6) alcanzaron un desarrollo semejante o mayor al de las otras especies, exceptuando Ocotea (ANOVA, para altura $\mathrm{F}_{6-63}=17,45$; área foliar $\mathrm{F}_{6-63}=6,23$; y materia verde $F_{6-63}=8,12, P<0,01$ ).

\section{DISCUSION}

En Punta Lara las malezas producen anualmente más diásporas que las plantas nativas. Debido a su alta densidad poblacional y productividad por unidad de área, Ligustrum es la planta ornitócora que más frutos y semillas forma en la selva, lo que explica el elevado número de plántulas que se observan. Rubus es muy eficiente en su reproducción porque con pocas flores produce muchas semillas (la mitad de sus flores dan frutos maduros y cada uno es multiseminado). Esta producción eficiente de diásporas implica que más recursos pueden ser asignados a otras funciones que compiten con la reproductiva (Bazzaz \& Ackerly 1992). De hecho, y como es frecuente en Rubus (Oleskevich et al. 1996), R. ulmifolius presenta un vigoroso crecimiento vegetativo y es la única de las especies estudiadas que se propaga asexualmente, mediante guías cuyos extremos enraízan y originan nuevas plantas.

Varias de las especies nativas logran pocos frutos en relación al esfuerzo invertido en producir flores (Ocotea, Allophylus y Blepharocalyx), y las que alcanzan una buena relación (Rapanea y Citharexylum) igualmente producen menos semillas que las exóticas. No obstante, es común que en muchas plantas, y en particular en las arbóreas, se observe una baja relación fruto/ flor (Stephenson 1981, Sutherland \& Delph 1984, Charlesworth 1989). Además, la producción de flores sobrantes no debe considerarse como una simple pérdida de recursos, habiéndose propuesto varias ventajas funcionales para las flores que no fructifican (Stephenson 1981, Sutherland \& Delph 1984, Ehrlén 1991).

Tanto las malezas como tres especies indígenas (Allophylus, Blepharocalyx y Rapanea) parecen 
tener asegurada una buena dispersión de las diásporas que forman. En este sentido la fenología reproductiva de las primeras podría haberlas favorecido, ya que presentan los frutos en momentos de baja oferta de la flora nativa. Si bien los frutos de Ligustrum constituyen un alimento de menor calidad que los de otras plantas, maduran en una época (pleno invierno) en que los recursos alimenticios escasean, a tal punto que la frecuencia de algunas especies de aves en la reserva aumenta durante su fructificación (Montaldo 1993)

La situación es diferente para las dos restantes especies nativas. Los frutos de Ocotea tienen características (tamaño grande, color verde, alto contenido en lípidos, semilla mayor a $10 \mathrm{~mm}$ ) que indican que son consumidos por aves marcadamente frugívoras (Howe 1986). Aunque la llegada de las malezas no afectó la situación de Ocotea en este sentido, ya que la fructificación no es coincidente, se observó baja eficiencia en su elenco dispersor. La no dispersión de las semillas por los frugívoros ocurre porque son destruídas antes de ser tragadas o en el aparato digestivo, o porque son descartadas antes de la ingestión del fruto ("predadores de pulpa", e.g., Jordano1992; como ocurre con Thraupidae, ver también Levey 1987). Sin embargo, dicho fenómeno puede producirse también por manejo deficiente del fruto por aves que lo tragan entero (el fruto se les "cae", observación personal), como es el caso de Turdus spp. que habitualmente es un consumidor "legítimo" de frutos (Snow 1971) porque dispersa las semillas (e.g., Sorensen 1984, Montaldo 1993). El rol de un frugívoro no depende solamente de su morfología y comportamiento, sino también de las características del fruto de la planta objeto de la interacción (Jordano 1992). En Ocotea el tamaño y la firmeza de la pulpa limitan el elenco dispersor a aves de fauces grandes (cf. Wheelwright 1985a, Levey 1987) y se desaprovechan muchos frutos por la baja relación tamaño del fruto/ tamaño del pico (cf. Snow \& Snow 1988) de los consumidores existentes en el área (Turdus spp.).

En cuanto a Citharexylum, sus semillas son sólo ocasionalmente dispersadas por las dos especies de Turdus y, además, sufre la predación intensa de Columba picazuro, cuyo potente sistema digestivo (Ziswiler \& Farner 1972) destruye la mayor parte de las semillas ingeridas. Esta especie de Columba también destruye gran cantidad de semillas de Ligustrum (ninguna de ellas sobrevive, Montaldo, datos no publicados), pero la producción de semillas de dicha maleza es muy grande. Por otra parte, muchos de los frutos de Citharexylum se deshidratan sobre las plantas como consecuencia de no ser removidos por las aves, probablemente debido a la competencia por dispersores (Wheelwright 1985b, Knight 1986) ejercida por Ligustrum, que superpone en gran medida su fructificación y tiene tres dispersores muy activos. Ligustrum tiene características que indican una baja especialización en el requerimiento de dispersores (frutos negros y de tamaño pequeño, semillas chicas, fructificación abundante y maduración sincrónica) (e.g., Murray 1987 y referencias en éste), mientras que Citharexylum es menos generalista en tal sentido. Pese a su pobre composición (cf. Moermond \& Denslow 1985, Herrera 1987) y menor relación pulpa/ semilla (cf. Moermond \& Denslow 1985, Howe 1986 y referencias en éstos), los frutos de Ligustrum son preferidos por las aves frugívoras cuando coexisten con los de Citharexylum. En esta especie la pulpa firme está fuertemente adherida al endocarpo (a diferencia de la de Ocotea, cuyo fruto es muy buscado por Turdus spp.), lo que haría más difícil su aprovechamiento y eleva el tiempo de pasaje de las semillas por el tracto de las aves. Dicho factor es crítico para determinar preferencias de frutos en Turdus spp., ya que Sorensen (1984) demostró que estas aves favorecen los que ofrecen un balance más favorable entre la energía obtenida de la pulpa y el tiempo de pasaje de la parte indigestible, básicamente las semillas.

Además de ser la especie que más semillas dispersa por episodio reproductivo, Ligustrum tiene también ventajas debidas al alto poder germinativo de sus semillas y a la capacidad de supervivencia de sus plántulas. De hecho, algunos sitios de la selva de Punta Lara parecen verdaderos almácigos de la especie exótica (observación personal). Esta abundancia de plántulas de Ligustrum seguramente interfiere con las especies nativas. Si bien tienen semillas con un poder germinativo superior al $50 \%$ en todos los casos estudiados y sus plántulas pueden crecer normalmente, el principal riesgo que deben afrontar para establecerse es la competencia con las plantas y/ o plántulas vecinas (Fenner 1985). En lo que hace a Rubus, cuyas grandes masas ocupan los claroso abras de la selva, es una especie pionera que se instala y coloniza por clonación sitios disturbados (Jordano 1982). Sus semillas germinan en bajo porcentaje en el primer año, pero muchas permanecen viables en el suelo. Como ocurre con otras especies del género (Marcuzzi \& Fernández de Martínez 1993, Oleskevich et al. 1996), las condiciones que estimulan a la semilla para salir de la fase de dormancia estarían asociadas con disturbios (incremento de luz y temperatura). Sus plántulas son muy agresivas por tener rápido cre- 
cimiento, elevada área foliar y hábito de apoyarse sobre otras plantas, con las que interfiere en su desarrollo. Esta maleza competiría exitosamente con las especies que, por tener mecanismos de dormancia regulados en forma semejante, requieren para germinar de la formación de claros en la selva (Vázquez-Yanes \& Orozco-Segovia 1993). Sin embargo, aún cuando haya alcanzado gran biomasa, Rubus puede ver limitado su crecimiento e incluso ser desplazada por la competencia interespecífica ejercida por el desarrollo de un estrato arbóreo superior (Jordano 1982, Oleskevich et al. 1996, observación personal).

Se cree que aunque la gran masa de agua del Río de la Plata actúa como un elemento compensador de factores climáticos y edáficos y permite el desarrollo de una selva subtropical en una región de clima templado (Cabrera \& Dawson 1944), no por ello la selva deja de encontrarse en una situación de marginalidad ecológica, con sus problemas derivados. Por cierto, el presente estudio permitió comprobar que hay especies nativas que tienen restricciones reproductivas: Citharexylum y Ocotea carecen de dispersores adecuados, situación análoga a la de los bosques del sur de Chile, donde algunas plantas no tendrían sus dispersores originales (Armesto et al. 1987). En el caso de Citharexylum, la superposición en la oferta de frutos con Ligustrum empeora la situación de esta especie nativa. La falta de mejores dispersores para Citharexylum y Ocotea en Punta Lara sería una manifestación del carácter marginal y relictual de esta selva, ya que se conocen consumidores no presentes en el área, pero sí en latitudes más septentrionales (Ridley 1930 , Moermond \& Denslow 1985). Igual significado tendría el hecho de que el elenco de aves frugívoras de Punta Lara sea reducido (únicamente ocho especies consumen los frutos de las plantas en estudio). Por ejemplo para Allophylus, Foster (1987) reportó 26 consumidores diferentes en una localidad selvática del Paraguay (ca. $27^{\circ} 11^{\prime} \mathrm{S}$ ). En Argentina, tanto la diversidad específica de la selva ribereña (Cabrera 1976) como la avifauna potencialmente dispersora de los vegetales (Narosky \& Yzurieta 1987) disminuyen marcadamente en el sentido norte-sur. Asimismo, y dado el patrón reproductivo observado en Punta Lara, se estima probable que antes de la llegada de las plantas exóticas (fundamentalmente de Ligustrum) ocurriesen desplazamientos estacionales (por ejemplo en invierno) de la avifauna frugívora hacia zonas más favorables.

Es de esperar que limitantes parecidas a las detectadas en la dispersión de algunas especies nativas ocurran en otras etapas de su ciclo de vida. Si bien en muchos casos la disponibilidad de polen compatible no parece ser la principal limitante del cuajado de frutos (Charlesworth 1989), es uno de los factores que restringen el éxito reproductivo de las plantas superiores (Feinsinger 1987, Whelan \& Goldingay 1989, Medan 1993). Entonces el bajo porcentaje de fructificación de Ocotea, Allophylus y Blepharocalyx podría deberse a escasez de polinizadores adecuados en esta latitud o adversidades durante la floración (por ejemplo temporales, con fuertes vientos que soplan desde el Río de la Plata y abaten muchas flores de Allophylus, observación personal). Apoyando este supuesto, en 1997 Allophylus produjo abundantes frutos maduros en el Parque Nacional El Palmar de Colón (provincia de Entre Ríos), donde la selva está en una latitud más favorable $\left(31^{\circ} 55^{\prime} \mathrm{S}\right)$ (Montaldo, datos no publicados), mientras que en Punta Lara su fructificación fue tan pobre como en los años anteriores (observación personal). También la importante colonización alcanzada por las malezas pudo introducir una distorsión en el ambiente polínico de la comunidad (competencia por polinizadores, modificaciones en la composición relativa de dicho gremio, entrega de polen inadecuado, etc., Waser 1983, Feinsinger 1987, McLernon et al. 1996), que resultó perjudicial para las plantas nativas. Se sabe que la introducción de malezas puede generar una interacción competitiva, que se da en forma más marcada en ambientes severos, donde las poblaciones de polinizadores son menores (Rathcke 1983). Este sería el caso de la selva de Punta Lara, en la que varias especies encuentran el límite sur de distribución (Cabrera \& Dawson 1944, Cabrera 1976).

$\mathrm{Si}$ bien se observaron algunas ventajas reproductivas para las plantas exóticas, es probable que otros factores, reproductivos o de otra índole (vulnerabilidad intrínseca del sistema nativo, ausencia de predadores naturales de las malezas, agresividad de las mismas: por ejemplo capacidad de multiplicarse vegetativamente y rápido crecimiento en $R u b u s$, etc.), sean responsables del deterioro actual de la selva de Punta Lara, aún en mayor medida que los hasta ahora estudiados. Aunque el presente estudio es una contribución parcial, dada la virtual falta de individuos de reemplazo en la flora autóctona y al mal estado que presentan muchos de sus ejemplares (observación personal), puede concluirse que únicamente medidas urgentes de manejo podrán evitar la pérdida de este relicto selvático biogeográficamente valioso. En la actualidad el mismo tiene ya características de un bosque mixto y, de no mediar la intervención del hombre, en un corto tiempo se convertirá en un bosque puro de Ligustrum lucidum, una especie originaria del lejano oriente. 


\section{AGRADECIMIENTOS}

A. Chiappe, G. Roitman, J. Loreti, J.J. Valla y L. Frank me dieron una valiosa ayuda en las tareas de campo. P. Cattaneo y su equipo hicieron el análisis químico de los frutos en el Departamento de Química Orgánica de la Facultad de Ciencias Exactas y Naturales, Universidad de Buenos Aires. Con R. Fraga consulté al elaborar el proyecto del trabajo y el manuscrito original se benefició con la lectura crítica de J.J. Valla y D. Medan, con quien intercambié opiniones a lo largo del estudio, y de dos revisores anónimos. Durante la investigación tuve una beca de perfeccionamiento para graduados de la Universidad de Buenos Aires, y el estudio en parte fue financiado con un subsidio de dicha institución.

\section{LITERATURA CITADA}

AOAC (1990) Official methods of analysis. 15th Edition Helrich K (Ed). Vol. 1. Association of Official Analytical Chemists. AOAC Inc. Arlington, Virginia. xxiv $+684 \mathrm{pp}$.

ARMESTO JJ, R ROZZI, P MIRANDA \& C SABAG (1987) Plant/frugivore interactions in South American temperate forests. Revista Chilena de Historia Natural 60: 321-336.

BARRETT SC \& CG ECKERT (1990) Current issues in plant reproductive ecology. Israel Journal of Botany 39: $5-12$.

BAZZAZ FA \& DD ACKERLY (1992) Reproductive allocation and reproductive effort in plants. En: Fenner $M$ (ed) Seeds: the ecology of regeneration in plant communities: 1-26. Commonwealth Agricultural Bureau International, Wallingford, Oxon.

CABRERA AL (1960) La selva marginal de Punta Lara. Ciencia e Investigación 16: 439-446.

CABRERA AL (1976) Regiones fitogeográficas argentinas. Enciclopedia Argentina de Agricultura y Jardinería. Segunda edición. Tomo 2, Fascículo 1. Editorial Acme, Buenos Aires. 85 pp.

CABRERA AL \& G DAWSON (1944) La selva marginal de Punta Lara en la ribera argentina del Río de La Plata. Revista del Museo de La Plata (Argentina) 5: 267-382.

CHARLESWORTH D (1989) Why do plants produce so many more ovules than seeds? Nature 338: 21-22.

DASCANIO LM \& SE RICCI (1988) Descripción florístico-estructural de las fisonomías dominadas por árboles en la Reserva Integral de Punta Lara (pcia. de Buenos Aires, República Argentina). Revista del Museo de La Plata (Nueva serie), Sección Botánica, 14: 191-206.

DEBUSSCHE M \& P ISENMANN (1990) Introduced and cultivated fleshy-fruited plants: consequences for a mutualistic Mediterranean plant-bird system. En: di Castri F, AJ Hansen \& M Debussche (eds) Biological invasions in Europe and the Mediterranean Basin: 399-416. Kluwer Academic Publishers, Dordrecht.

EHRLEN J (1991) Why do plants produce surplus flowers? A reserve-ovary model. American Naturalist 138: 918-933.

FEINSINGER, P (1987) Effects of plant species on each other's pollination: is community structure influenced. Trends in Ecology \& Evolution 2: 123-126.

FENNER, M (1985) Seed ecology. Chapman \& Hall, London. vii + 151 pp.

FISCHER KE \& CA CHAPMAN (1993) Frugivores and fruit syndromes: differences in patterns at the genus and species level. Oikos 66: 472-482.

FOSTER MS (1987) Feeding methods and efficiencies of selected frugivorous birds. Condor 89: 566-580.

FUENTES M (1995) How specialized are fruit-bird interactions? Overlap of frugivore assemblages within and between plant species. Oikos 74: 324-330.

HERRERA CM (1985) Determinants of plant-animal coevolution: the case of mutualistic dispersal of seeds by vertebrates.Oikos 44: 132-141.

HERRERA CM (1987) Vertebrate-dispersed plants of the Iberian Peninsula: a study of fruits characteristics. Ecological Monographs 57: 305-331.

HERRERA CM (1995) Plant-vertebrate seed dispersal systems in the Mediterranean: ecological, evolutionary, and historical determinants. Annual Review of Ecology and Systematics 26: 705-727.

HERRERA CM \& P JORDANO (1981) Prunus mahaleb and birds: the high-efficiency seed dispersal system of a temperate fruiting tree. Ecological Monographs 51: 203-218.

HEYWOOD VH (1989) Patterns, extents and modes of invasions by terrestrial plants. En: Drake JA, HA Mooney, F di Castri, RH Groves, FJ Kruger, M Rejmánek \& M Williamson (eds) Biological invasions. A global perspective: 31-55. SCOPE 37. J Wiley \& Sons, Chichester.

HOWE HF (1986) Seed dispersal by fruit-eating birds and mammals. En: Murray DR (ed) Seed dispersal: 123 189. Academic Press Australia, Sydney.

JORDANO P (1982) Migrant birds are the main seed dispersers of blackberries in southern Spain. Oikos 38: 183-193.

JORDANO P (1987) Patterns of mutualistic interactions in pollination and seed dispersal: connectance, dependence asymmetries, and coevolution. American Naturalist 129: 657-677.

JORDANO P (1992) Fruits and frugivory. En: Fenner M (ed) Seeds: the ecology of regeneration in plant communities: 105-156. Commonwealth Agricultural Bureau International, Wallingford, Oxon.

JORDANO P (1995a) Angiosperm fleshy fruits and seed dispersers: a comparative analysis of adaptation and constraints in plant-animal interactions. American Naturalist 145: 163-191.

JORDANO P (1995b) Frugivore-mediated selection on fruit and seed size: birds and St. Lucie's cherry, Prunus mahaleb. Ecology 76: 2627-2639.

KLIMAITIS JF \& F MOSCHIONE (1987) Aves de la Reserva Integral de Selva Marginal de Punta Lara y sus alrededores. Ministerio de Asuntos Agrarios de la Provincia de Buenos Aires, Buenos Aires. 145 pp. 
KNIGHT RS (1986) Fruit displays of indigenous and invasive alien plants in the South-Western Cape. South African Journal of Botany 52: 249-255.

LEDESMA NR (1953) Registro fitofenológico integral. Meteoros 3: 81-96.

LEVEY DJ (1987) Seed size and fruit-handling techniques of avian frugivores. American Naturalist 129: 471 485.

MARCUZZI, IM \& EA FERNANDEZ DE MARTINEZ (1993) Estudio de la dormición en semillas de frambueso (Rubus idaeus L.). Phyton 54: 139-147.

MATEUCCI SD \& A COLMA (1982) Metodología para el estudio de la vegetación. Serie de biología, Monografía $\mathrm{N}^{\circ} 22$, Secretaría General de la Organización de los Estados Americanos, Washington. vi + $168 \mathrm{pp}$.

MCLERNON SM, SD MURPHY \& LW AARSEN (1996) Heterospecific pollen transfer between sympatric species in a midsuccessional old-field community. American Journalof Botany 83: 1168-1174.

MEDAN D (1993) Breeding system and maternal success of a perennial hermaphrodite, Discaria americana (Rhamnaceae). New Zealand Journal of Botany 31: 175-184.

MONTALDO NH (1993) Dispersión por aves y éxito reproductivo de dos especies de Ligustrum (Oleaceae) en un relicto de selva subtropical en la Argentina. Revista Chilena de Historia Natural 66: 75-85.

MOERMOND TC \& JS DENSLOW (1985) Neotropical avian frugivores: patterns of behavior, morphology and nutrition, with consequences for fruit selection. En: Buckley PA, MS Foster, ES Morton, RS Ridgely \& FG Buckley (eds) Neotropical Ornithology: 865897. Ornithological Monographs 36. American Ornithologists' Union, Washington.

MOORE RP (1985) Handbook on tetrazolium testing. International Seed Testing Association, Zurich. 99 pp.

MURRAY KG (1987) Selection for optimal fruit-crop size in bird-dispersed plants. American Naturalist 129: 18-31.

NAROSKY T \& D YZURIETA (1987) Guía para la identificación de las aves de Argentina y Uruguay. Asociación ornitológica del Plata, Buenos Aires. 345 pp.

NOBLE IR (1989) Attributes of invaders and the invading process: terrestrial and vascular plants. En: Drake JA, HA Mooney, F di Castri, RH Groves, FJ Kruger, M Rejmánek \& M Williamson (eds.) Biological invasions. A global perspective: 301-313. SCOPE 37. J Wiley \& Sons, Chichester.

OLESKEVICH C, SF SHAMOUN \& ZK PUNJA (1996) The biology of Canadian weeds. 105. Rubus strigosus Michx., Rubus parviflorus Nutt., and Rubus spectabilis Pursh. Canadian Journal of Plant Science 76: 187 201.

PHELPS WH Jr. \& R MEYER DE SCHAUENSEE (1979) Una guía de las aves de Venezuela. Gráficas Armitano, Caracas. $x x v+484 \mathrm{pp}$.

RATHCKE B (1983) Competition and facilitation among plants for pollination. En: Real L (ed) Pollination biology: 305-329. Academic Press, New York.

REICHARD SH \& CW HAMILTON (1997) Predicting invasions of woody plants introduced into North America. Conservation Biology 11: 193-203.
REJMANEK M \& DM RICHARDSON (1996) What attributes make some plant species more invasive? Ecology 77: 1655-1661.

RIDLEY HN (1930) The dispersal of plants throughout the world. L. Reeve, Ashford, Kent, xx+744 pp.

SICK H (1986) Ornitologia brasileira, uma introduçao. Segunda ediçao. Vol. 2. Editora Universidade de Brasilia, Brasilia. viii $+347 \mathrm{pp}$.

SIEGEL S (1975) Estadística no paramétrica. Editorial Trillas, México. $346 \mathrm{pp}$.

SNOW DW (1971) Evolutionary aspects of fruit-eating by birds. Ibis 113: 194-202.

SNOW BK \& DW SNOW (1988) Birds and berries. T \& AD Poyser, Calton. 268 pp.

SOKAL RR \& FJ ROHLF (1969) Biometry. The principles and practice of statistics in biological research. WH Freeman \& Co., San Francisco. xxi +776 pp.

SORENSEN AE (1984) Nutrition, energy and passage time: experiments with fruit preference in European Blackbirds (Turdus merula). Journal of Animal Ecology 53: 545-557.

STEPHENSON AG (1981) Flower and fruit abortion: proximate causes and ultimate functions. Annual Review of Ecology and Systematics 12: 253-279.

SUTHERLAND S \& LF DELPH (1984) On the importance of male fitness in plants: patterns of fruit-set. Ecology 65:1093-1104.

TIMMINS SM \& PA WILLIAMS (1991) Weed numbers in New Zealand's forest and scrub reserves. New Zealand Journal of Ecology 15: 153-162.

VAN DER PIJL L (1982) Principles of dispersal in higher plants. Third Edition.Springer-Verlag, Berlin. $\mathrm{x}+$ $215 \mathrm{pp}$.

VAZQUEZ-YANES C \& A OROZCO-SEGOVIA (1993) Patterns of seed longevity and germination in the tropical rainforest. Annual Review of Ecology and Systematics 24: 69-87.

WASER NM (1983) Competition for pollination and floral character differences among simpatric plant species: a review of evidence. En: Jones CE \& RJ Little (eds) Handbook of experimental pollination biology: 277293. Van Nostrand Reinhold, New York.

WHEELWRIGHT NT (1985a) Fruit size, gape width, and the diets of fruit-eating birds. Ecology 66: 808-818.

WHEELWRIGHT NT (1985b) Competition for dispersers, and the timing of flowering and fruiting in a guild of tropical trees. Oikos 44: 465-477.

WHEELWRIGHT NT \& CH JANSON (1985). Colors of fruit displays of bird-dispersed plants in two tropical forests. American Naturalist 126: 777-799.

WHELAN RJ \& RL GOLDINGAY (1989) Factors affecting fruit-set in Telopea speciosissima (Proteaceae): the importance of pollen limitation. Journal of Ecology 77: 1123-1134.

WHITE DW \& EW STILES ( 1992) Bird dispersal of fruits of species introduced into eastern North America. Canadian Journal of Botany 70: 1689-1696.

ZISWILER V \& DS FARNER (1972) Digestion and the digestive system. En: Farner DS \& JR King (eds) Avian Biology. Vol. 2: 343-430. Academic Press, New York. 